\title{
A Dynamic Sequence Model of Information Sharing Processes in Virtual Teams
}

\author{
Aaron Schecter \\ University of Georgia \\ aschecter@uga.edu
}

\author{
Noshir Contractor \\ Northwestern University \\ nosh@northwestern.edu
}

\begin{abstract}
Sharing information is a critical component of virtual team functioning. While prior research has identified the motivations for and the structure of information sharing, there has been little emphasis on the dynamic patterning of sharing behavior. In this study, we focus on the process of information sharing, namely the sequence and timing of individual decisions during a virtual team task. Further, we argue that sharing behaviors can be categorized into a finite number of approaches. We propose a temporal, eventbased model to uncover the behavioral and cognitive factors that influence information sharing. With a sample of 600 participants organized into thirty ad hoc virtual teams, we demonstrate significant heterogeneity in sharing propensities. Our study makes two contributions to the extant literature. First, we extend theories regarding the motivation and structure of information sharing. Second, we make a broader methodological contribution with the application of a latent-class relational event model.
\end{abstract}

\section{Introduction}

Knowledge is a resource that is vital to organizational functioning and performance [1]. To build organizational knowledge, the resources of individuals, e.g. information or expertise, must be efficiently combined. Essentially, organizations function as "open social systems that must process information...to accomplish internal activities, to coordinate diverse activities, and to interpret the external environment” [2]. Following Nonaka (1994), we define information as "a flow of messages, and knowledge is created by the very flow of information" [3]. From this perspective, for an organization to generate the knowledge it requires there must be a sufficient flow of information among members of the group so that expertise can be effectively integrated [4]. This process is particularly relevant in teams, which are often the building blocks of organizations [5].
Information functions as a resource of the team, and teams convert their available resources into actions and or products [1].

The success of face-to-face work teams [6] and virtual teams [7] hinges on the ability of individuals to share information amongst themselves, and subsequently synthesize it in a meaningful way [4]. Indeed, a high level of information sharing indicates that the requisite item was provided in a timely manner throughout the team's work cycle, "thereby enabling groups to reach higher quality solutions that could be reached by any one individual" [6]. Conversely, a failure to provide internally held information can impede team performance in a number of ways, such as limiting innovation or creativity [8], [9]. A fundamental aspect of this process is the willingness of individuals to frequently provide teammates with relevant information. When individuals share information, they broaden the information space available to the team, i.e. illuminating relevant facts, as well as enhance the potential for meaningful solutions or outcomes [10]. Through frequent communication and information sharing, team members also develop an awareness of where expertise is located, who is an accurate source of knowledge, and who has access to other sources of information [11]-[13]. This behavior over time allows for more effective sharing in the future, and subsequently higher levels of performance [12].

Because information sharing is an integral component of team success, it is important to understand how information is actually transferred from one individual to another. In particular, we consider the case of virtual teams that by design are often composed of multiple distributed units [14], [15]. This separation could be due to geographic separation, temporal asynchrony, or language and cultural barriers. These conditions tend to force a reliance on multiple virtual means of communication to bridge the divide [16]. As such, virtual teams are distinct from face-to-face teams in the way that information is shared and processed. In particular, when virtual channels are implemented, individuals can be more thoughtful and selective with the information that they share. Accordingly, more unique knowledge can be transmitted [7]. Conversely, a 
lack of face-to-face contact can stymy an open flow of communication regarding that information, leading to slower synchronization and processing [4].

When individuals do choose to share information and collaborate in a virtual team, they must determine where to route that information so that it reaches the intended recipient. However, they may not possess the meta-knowledge necessary to send information directly [13], [17]. Thus, we expect members to rely on a set of heuristics to make their decisions. In other words, instances of individuals sharing information are driven by a mix of behavioral trends and cognitive factors. Further, the role each of these effects has on a person's actions is unique to that individual. For instance, an individual may choose to share information with a frequent informal communication partner. On the other hand, a separate individual may try to guess who the most central or active person is, and send information to that actor. To uncover these effects and their influence on decisionmaking, we shift our focus to how the process of transferring information occurs. Namely, we consider how contextual changes or trends in behavior can shape sharing tendencies. Accordingly, we propose a processoriented perspective where propensities to share information are formed dynamically as functions of both psychological constructs and explicit behavioral patterns [18], [19]. A process-oriented approach treats information sharing as a series of events, i.e. the transfer of knowledge from one individual to another, that unfold over time [20]. As such, individuals follow information sharing trajectories, where their propensities to act are continuously updated as new events occur and the context shifts.

The purpose of this study is to uncover the behavioral and cognitive factors that influence information sharing, and to determine how these mechanisms combine to predict individual decisions. To address this research objective, we argue for a dynamic perspective on information sharing and derive three types of mechanisms that should influence decision making. Second, we introduce a novel methodology relational event models with latent behavioral classes [21], [22] - for identifying the strategies employed by individuals in a simulated virtual team environment. With a sample of 600 participants organized into thirty teams, we demonstrate significant heterogeneity in sharing propensities among individuals. In particular, we identify two distinct sets of criteria for decision making with qualitative differences. Our study makes two contributions to the extant literature on virtual team coordination processes. First, we extend theories regarding why individuals share information to include with whom and when that information is transmitted. To do so, we adopt a processual lens of sharing behavior.
Second, we make a methodological contribution by introducing a model to identify decision-making strategies made over time, and to account for heterogeneity across individuals.

\section{Conceptual Development}

We draw on prior literature to build a picture of the information sharing process for members of virtual teams. In particular, we focus on ad hoc virtual teams, or those without significant development of transactive memory systems [23]-[25]. This distinction is important because individuals who choose to share information must rely on a set of behavioral patterns and experiences, rather than meta-knowledge of who knows who and who knows what [17]. First, we argue for a dynamic perspective on information sharing in additional to a more conventional structural approach. To account for temporality, we propose an event-based framework for analysis. Second, we delineate the behavioral and cognitive factors that contribute to an individual's sharing decisions. Each measure is based on prior activities and the virtual team context. We posit that these factors are combined in a finite number of ways to produce decision-making criteria that individuals use when sharing information.

\subsection{Information Sharing Dynamics}

Given the implications of information sharing for performance in organizations, it is important to understand how individuals actually engage in the process of transferring knowledge [26]. Members of an organization may be more or less likely to share information depending on its nature and their attitudes about that information (Constant, Kiesler, \& Sproull, 1994). Indeed, the likelihood of an individual contributing knowledge to an organization or community can be affected by several factors, including social capital, group norms, and feelings of self-efficacy [27]-[29]. In online communities, a tendency towards reciprocity, both direct and indirect, can motivate an individual to contribute [30]. Further, as [31] point out, the motivations for exchanging knowledge vary based on how information is defined by the organization; when information is an object, it is exchanged through interactions with others. In this case, individuals are motivated by self-interest, e.g. gaining rewards or fulfilling obligations.

While there are various potential motivations, the actual factors underlying behavior are only expressed over time as people make decisions and engage in group processes. Information sharing occurs during the process of virtual team coordination, or the 
interactions between individuals that manage resources and expertise dependencies. Coordination plays a vital role in effective information sharing by identifying who knows what, who needs what, and mobilizing the available resources [17]. The coordination of expertise in a team has both structural and temporal elements. The structural or configural view of coordination posits that interactions follow distinct patterns, and that there is a relationship between these structures and organizational outcomes [32], [33]. To determine how collaboration is structured, it is useful to represent the team as a network with individuals as nodes and links between the nodes signifying joint taskwork or coordination. From this perspective, an individual may choose to share information based on their local network, i.e. those they have ties with, or may direct information towards central actors. Further, individuals may delineate between informal communication networks and expertise networks, and share information with those whom they communicate with on a more general level [34].

Coordination is temporal in that structures are not constant over time; rather, they emerge as a result of repeated actions [33]. In the context of information sharing, the decision to transmit information from one individual to another is a distinct activity that occurs at a specific point in time, and occurs with the overarching structure providing context. The temporal perspective shifts the focus from what structures facilitate information sharing, to how individuals actually engage in sharing. Accordingly, events occur in the context of the coordination network, and can subsequently reshape that network.

To unveil the temporal patterns of information sharing, we propose an event-based framework for understanding the individual decision process. An event-based approach to understanding behavior places the focus on the interactions that occur and how they unfold over time [20], [35]. In this way, patterns we identify represent generative mechanisms that link the occurrence of one event to a subsequent action, thus driving organizational change [19]. The collection of generative mechanisms that shape interaction processes for an individual constitute their strategy for selecting targets and disseminating information. This generative structure defines how events can transpire, even if the exact internal structure of activities varies[37]. Thus, individuals can be compared by the commonalities in the pattern and sequencing of information sharing actions.

\subsection{Factors Influencing Sharing Behavior}

We proceed to describe three types of mechanisms that drive information sharing: tendencies derived from the communication network, tendencies derived from prior sharing behavior, and tendencies derived from individual awareness of the situation. The influence each of these factors has on the propensity to share information will be a characteristic of an individual and represent the norms governing their sharing practices [26]. By understanding how past behaviors influence future actions, it is possible to uncover the latent tendencies of each member of the virtual team. Essentially, each individual falls into a category that describes the overall trends in their actions; though these classes cannot be observed directly, the categories can be inferred by delineating distinct action patterns [37], [38]. As such, we expect that every individual will have a unique behavioral "signature" that describes their approach to transferring knowledge throughout the team. This signature determines how sharing partners are selected, as well as how frequently and at what time information is shared.

2.1.1 Communication Network. Communication network effects describe the impact of informal communication networks on the tendencies for individuals to share information [39]. Informal communication patterns are distinct from formal task interdependencies, and form as a result of attempts to coordinate [34]. We focus on four generative mechanisms of information sharing based on network structure: tie strength, activity, preferential attachment, and transitive closure. The first network mechanism is tie strength. Having strong ties between members can reduce the cost associated with transferring information [39], [40], and in general the degree of interconnection in the organization should promote greater sharing of knowledge [15]. Frequent contact will make sharing information faster and more efficient, but at the cost of tie maintenance [41]. Consequently, individuals may rely on a dyadic strategy to promote ease of transferal, but others may prefer to rely on weaker, more ephemeral ties [42]. Our second mechanism, activity, describes the tendency for individuals who are most active in the communication network to also share the most information. Essentially, one who has the greatest number of connections will have greater opportunity to transfer information due to their broader potential range [39]. Alternatively if the third mechanism, preferential attachment, drives information sharing, then an individual would send information to recipients who are highly central in the communication network [43]. This mechanism could come about because of simple attraction - i.e. one person becomes focal - or because a central individual may be viewed as having better awareness of who needs what intelligence [13]. Finally, individuals who employ a strategy based on transitive closure will choose to send information to targets with whom they communicate through a third-party broker 
[20], [34]. In other words, rather than pass the information through a middleman, they send it directly to the intended recipient. However, there is a caveat to the preferential attachment and transitivity mechanisms; individuals may not be able to determine who is actually central or who is one degree separated from themselves. Thus, if we find that either mechanism is influential, it may only be a behavioral trend, not a concerted decision.

2.1.2 Sharing Behavior. Sharing behavior effects describe the impact of prior sharing patterns on future transmission decisions. These interactions are distinct from other forms of communication in that they are directly related to technical details needed by the team [33], [34]. These explanations include prior transference behavior, sharing reciprocity, total sharing activity, and sharing popularity of the recipient. Prior transference behavior encapsulates the tendency towards inertia, where one individual tends to send information to the same set of partners. Alternatively, sharing reciprocity describes a tendency for individuals to share information with others who have previously sent them information [30]. Both inertia and reciprocity are indicative of a reliance on well-developed pathways and existing social capital [27]. The mechanism of prior sharing activity encapsulates the tendency for an individual to maintain or expand the prior rate of sharing behavior. For example, an agent may determine that an optimal strategy is to share information as frequently as possible. Finally, the fourth mechanism of sharing is a focus on transferring information to the individuals who are most frequently receive information. This effect describes the tendency for information to flow to individuals who are focal in the overall sharing process. Like the preferential attachment mechanism, popular targets for information may be viewed as having a better understanding of where information belongs, and thus can route intelligence more effectively. This mechanism also comes with the same qualifier as preferential attachment; though we may observe the behavior, it is difficult to determine cognition.

2.1.3 Situational Awareness. The final set of generative mechanisms we consider are based on an individual's awareness of the task parameters and the team's progress towards their goal. Specifically, we focus on two factors, information redundancy and time constraints, though others are possible depending on the nature of the task. An individual who is aware of redundancy will decide to pass information based on whether that intelligence is unique to the recipient or not. Though some redundancy is necessary - e.g. sending reminders - we expect that an actor who is highly aware of the team's status will avoid sharing redundant information. The second mechanism is awareness of time constraints, which encapsulates an individual's reaction to the task deadline. An individual who is more aware may act proactively and share information quickly after receiving it.

Alternatively, someone who is not motivated by time constraints will pass information along at random intervals, or perhaps even wait until the last minute to disseminate information.

\section{Methods}

\subsection{Data}

We collected data through a series of experiments where participants had to complete an information sharing task in a virtual team environment. The sample is composed of 600 unique individuals organized into thirty virtual teams. Participants were recruited at a Midwestern US university and participated in this study in exchange for either research credit or \$35. Individuals reported to a laboratory in groups of twenty, forming a single virtual team, and each group was conducted in a separate two-hour session. The session consisted of pre- and post-game surveys, a twenty-minute practice mission, and a forty-minute performance mission. For the purpose of this study we only consider data from the performance mission component. Participants were randomly assigned to one of four functionally equivalent units within the virtual organization, and to a specific role within their component. Each participant was seated at an individual workstation, and performed the task using a laptop computer.

In each experimental session, the teams participated in a computer-based simulation game that entailed guiding a humanitarian aid convoy through dangerous territory. Each of the four units were responsible for clearing targets within one quadrant of the game map. The convoy could not progress through the map until the obstacles in its path were eliminated. In order to clear a target on the map, one member of the team - a reconnaissance officer - had to identify an obstacle by flagging the correct portion of the grid. If the recon officer chose an incorrect space on the grid, the target would remain. There are two types of obstacles: insurgents and IEDs. A field specialist and recon officer were assigned to one type of task or another, and could not identify the other type on the map. Once the obstacle is correctly identified, a field specialist on the component team could then neutralize the threat. All four units also had a designated navigator who would help determine the path of the convoy. 
In total, all four quadrants have thirty-two threats located inside them. However, each five-person unit is only given the locations of eight of those targets. The remaining twenty-four locations are evenly distributed among the other three groups. Each unit therefore possessed thirty-two pieces of intelligence, but twenty-four needed to be distributed to the other fifteen members of the team. The intelligence was provided randomly to participants on a sheet of paper at the beginning of the study. While each unit had to clear their own obstacles, the participants were judged on how far the convoy was moved. As such, there was a clear performance incentive to share the pieces of information that the individuals did not need. All information had to be shared through direct communication; there was no distinct game feature for sharing intelligence.

Individual participants were given a moniker based on their functional unit and the task they were assigned (reconnaissance officer, field specialist, or leader). However, participants did not know a priori which other person needed the information in their possession; the names given to individuals in the game did not specify the type of target they were able to eliminate. Further, it was up to the individual participants to determine which functional unit was responsible for a particular coordinate. Thus, the correct recipient of each piece of intelligence was not immediately obvious to actors in the game.

Participants wore headsets and communicated with one another through Skype. Team members were allowed to choose between text-messaging through Skype chat or video calls with all other participants. We collected a full time-stamped transcript of all communication for each session, which provides us with data on who said what to whom at what time. This transcript was composed of both the text and audio information. Manual coders went through each line of communication and marked lines that contained pieces of intelligence. Further, the coders gave a unique identifier to each target, so the accuracy of the information sharing could be assessed. We provide a sample communication excerpt (with names simplified to Player 1 and 2) in Figure 1.

All aspects of the experimental set-up and task were held constant across sessions; variation in outcomes was a result of differences in participant behavior.

\subsection{Measures for Sharing Patterns}

To compute measures for each of our identified mechanisms, we converted our coded communication transcripts into relational event sequences. From these sequences, we computed two arrays, $U$ and $V$, which were weighted adjacency matrices with value at each point in time. The entry $(i, j)$ at time $t$ of $U(t)$ is represented as $u_{i j t}$, and is equal to the number of messages $i$ has sent $j$ up to time $t$. Likewise, the entry $(i, j)$ at time $t$ of $V(t)$ is represented as $v_{i j t}$, and is equal to the number of messages $i$ has sent $j$ up to time $t$ that contain coordinate information. Accordingly, $u_{i j t} \geq$ $v_{i j t}$ for all $i, j, t$. Using these arrays, we can compute statistics representing the mechanisms at every point in time, for every feasible pair of people. In Table 1 we list our variables and relevant formulae.

\subsection{Modeling}

In order to determine the factors that influence individual sharing decisions, we need a model with three key elements: (1) be able to capture the effect of each mechanism on the likelihood of sharing information; (2) be able to account for variability in the mechanisms over time; and (3) be able to identify heterogeneity in behavioral patterns. An appropriate framework for this problem is the relational event model (REM; [48], [49]. Relational events are single instances of an action involving a sender, receiver, and timestamp. For instance, a relational event may be a single line of text in a conversation or an edit to a software repository. A REM is built to determine the likelihood of a relational event, or realization of a network link, based on the sequence of events that have previously transpired. Relational event models combine the analytical techniques of event history modeling with the graphical or link-based representation used in social network analysis. In this way, REMs are an ideal choice for modeling the effect of generative mechanisms on behavior [46]. Further, the path dependency of the relational event model accounts for the continuously updating values of the mechanisms derived from event sequences.

\begin{tabular}{lllll}
\hline Time & Sender & Receiver & Message & Information \\
\hline 20:30:30 & Player 1 & Player 2 & I have info on a threat & N \\
20:30:42 & Player 2 & Player 1 & What is it? & N \\
20:31:07 & Player 1 & Player 2 & Two actually & N \\
20:32:26 & Player 1 & Player 2 & AFV, Cell J2, X: 431 Y: 138 & Y (ID: 48) \\
20:32:44 & Player 1 & Player 2 & RPG, Cell H2, X:186, Y: 153 & Y (ID: 21) \\
\hline
\end{tabular}

Figure 1. Sample coded communication excerpt 


\begin{tabular}{|c|c|c|}
\hline Variable Name & Description & Formula \\
\hline Prior & The number of messages exchanged between two & $u_{i j t}$ \\
\hline Communication & $\begin{array}{l}\text { individuals as a proportion of total } \\
\text { messages }\end{array}$ & $\overline{\sum_{k} u_{i k t}}$ \\
\hline Sender Activity & $\begin{array}{r}\text { The number of messages an individual has sent in } \\
\text { the past as a proportion of total messages }\end{array}$ & $x_{2}(i, j, t)=\frac{\sum_{k} u_{i k t}}{\sum_{l} \sum_{k} u_{l k t}}$ \\
\hline Receiver & The number of messages an individual has & $\sum_{k} u_{k j t}$ \\
\hline Popularity & $\begin{array}{l}\text { received in the past as a proportion of } \\
\text { total messages }\end{array}$ & $\overline{\sum_{l} \sum_{k} u_{k l t}}$ \\
\hline \multirow[t]{2}{*}{ Triadic Closure } & The number of messages sent to a target through & $\sum_{k} u_{i k t} u_{k j t}$ \\
\hline & $\begin{array}{l}\text { intermediaries as a proportion of all two- } \\
\text { paths }\end{array}$ & $\sum_{(a, b)} \sum_{k} u_{a k t} u_{k b t}$ \\
\hline \multirow[t]{2}{*}{ Prior Sharing } & The information shared from one individual to & $x_{5}(i, j, t)=\frac{v_{i j t}}{\nabla}$ \\
\hline & $\begin{array}{l}\text { another as a proportion of total } \\
\text { information sent }\end{array}$ & $\sum_{k} v_{i k t}$ \\
\hline \multirow[t]{2}{*}{ Reciprocal Sharing } & The information received from one individual as a & $v_{j i t}$ \\
\hline & proportion of total information received & $\sum_{k} v_{k i t}$ \\
\hline Sharing Activity & $\begin{array}{l}\text { The volume of information shared as a proportion } \\
\text { of total information shared }\end{array}$ & $x_{7}(i, j, t)$ \\
\hline \multirow{2}{*}{ Sharing Popularity } & The volume of information received as a & $\begin{array}{l}\sum_{l} \sum_{k} v_{l k t} \\
\sum_{k} v_{k j t}\end{array}$ \\
\hline & proportion of total information shared & $x_{8}(i, j, t)=\frac{2 k v_{k J t}}{\sum_{l} \sum_{k} v_{k l t}}$ \\
\hline
\end{tabular}

\section{Table 1. Key behavioral mechanisms for REM analysis}

To account for behavioral heterogeneity, we build on prior extensions to the core REM that uncover latent classes of actions [21], [22], [47]. We use these classes of actions to delineate distinct sets of decision-making approaches.

\subsection{Analysis of Behaviors}

To identify the unique patterns exhibited by the participants in our study, we used an unsupervised approach to determine the optimal combination of variables and groups. In particular, we fit the aforementioned model with a variety of parameter combinations - network terms only, prior sharing only, situational awareness only, pairwise combinations, and the full model - and a range of groups (i.e. $\mathrm{P}=1,2$, $3, \ldots)$. We assessed model quality using the loglikelihood, as well as the Bayesian Information Criterion (BIC) in order to avoid overfitting. The BIC is a measure of model fit that takes into account the number of parameters, relative to the number of observations. The best combination of parameters and groups will have the greatest log-likelihood and smallest BIC value. If these measures indicate that a multi-group solution is optimal (i.e. $\mathrm{P}>1$ ), then we may conclude that there are distinct sets of decision-making criteria that govern sharing behavior.

\section{Results}

Following our analysis procedure, we tested a variety of models with different sets of parameters and a range of groups. We identify the best fitting model as one with two groups and all parameters included. We thus conclude that there are two dominant trends in information sharing behavior. To determine what factors characterize these two approaches, we examine the parameter values for this best model. The results are presented in Table 3. Overall, 253 individuals were in Group 1 and 235 were in Group 2; we observed anywhere from 2 to 18 team members in either group, though most teams had a more even split.

From Table 3 we observe a number of key differences between the two criteria. To better illustrate the discrepancies, we take the differences between each of the parameter values across groups. Further, in a relational event model, $\exp \left(\beta_{11}-\beta_{21}\right)$ is equal to the relative rate at which group 1 will engage in an event compared to group 2, assuming the values of statistic 1 are the same. Thus, if this value is less than 1 , group 2 is much more likely to be influenced by that mechanism, and vice versa.

Individuals using the Group 1 approach demonstrate a tendency to share more information as they become more central, i.e. they are more active in the communication network $(\beta=6.56, p<0.001)$. In other words, these individuals share more as they communicate more. Individuals using this approach also tend to share information with others who are less central in the communication network $(\beta=-1.42, \mathrm{p}<$ $0.01)$. 


\begin{tabular}{|c|c|c|c|c|c|}
\hline & \multicolumn{2}{|c|}{ Model 1} & \multicolumn{2}{|c|}{ Model 2} & \multirow[b]{2}{*}{ Relative Rate } \\
\hline & Estimate & SE & Estimate & SE & \\
\hline Constant & -12.05 & $(0.07)^{* * *}$ & -9.45 & $(0.05)^{* * *}$ & \\
\hline Tie Strength & 0.78 & $(0.11)^{* * *}$ & 1.01 & $(0.11)^{* * *}$ & 0.79 \\
\hline Sender Activity & 6.56 & $(0.47)^{* * *}$ & -2.65 & $(0.42)^{* * *}$ & 9996.60 \\
\hline Receiver Popularity & -1.42 & $(0.45)^{* *}$ & 1.72 & $(0.50)^{* * *}$ & 0.04 \\
\hline Transitive Closure & 2.24 & $(0.24)^{* * *}$ & 0.94 & $(0.24)^{* * *}$ & 3.67 \\
\hline Prior Sharing & 3.38 & $(0.06)^{* * *}$ & 2.94 & $(0.06)^{* * *}$ & 1.55 \\
\hline Reciprocal Sharing & 0.65 & $(0.09)^{* * *}$ & 0.51 & $(0.09)^{* * *}$ & 1.14 \\
\hline Sharing Activity & 3.22 & $(0.13)^{* * *}$ & -0.05 & $(0.13)$ & 26.32 \\
\hline Sharing Popularity & 0.31 & $(0.17)$ & 0.34 & $(0.17)^{*}$ & 0.98 \\
\hline Redundancy & 0.02 & $(0.00)^{* * *}$ & 0.02 & $(0.00)^{* * *}$ & 1.00 \\
\hline Possession Time & 2.92 & $(0.08)^{* * *}$ & -3.12 & $(0.09)^{* * *}$ & 419.89 \\
\hline Number of Individuals & 251 & & 235 & & \\
\hline Log-Likelihood & $-18,093$ & & $-18,496$ & & \\
\hline
\end{tabular}

Table 3. REM results for two-group solution

Conversely, individuals in Group 2 share less as they become more central $(\beta=-2.65, \mathrm{p}<0.001)$, and they prefer to share information with highly central members $(\beta=1.72, p<0.001)$.

Both sets of individuals tend to share information with common third parties $(\beta=2.24, \mathrm{p}<$ $0.001 ; \beta=0.94, p<0.001$ ), though that behavior is much more common amongst those using approach 1 . In summary, the decision-making criteria differ in how network position impacts sharing propensities. Those applying the first approach share more as they become more central, and focus on transmitting intelligence to those who are more peripheral. On the other hand, those applying the second approach are more likely to share when they are less active in the network, and tend to route information to the most central members.

Individuals using the Group 1 approach have a strong tendency to share information along channels they have utilized in the past $(\beta=3.38, p<0.001)$. Those applying the Group 2 approach also follow this trend $(\beta$ $=2.94, \mathrm{p}<0.001$ ), though the effect is smaller. Both groups tend to be reciprocal in information sharing, though there is only a negligible difference. Additionally, individuals using the Group 1 approach are far more likely to share information when they are central in the sharing network $(\beta=3.22, p<0.001)$, i.e. they have transmitted a high volume in the past. Thus, unlike those using the Group 2 criteria, the first group tends to accelerate their rate of sharing as they share more. The key difference between the two decisionmaking criteria is the greater repetition of prior behavior observed in Group 1. We observe that both groups only exhibit a marginal tendency towards redundant communication, and there is no meaningful difference. Thus, individuals using either approach do not frequently share redundant information. However, the groups do differ on when they share, relative to the mission parameters. Individuals applying the Group 1 approach tend to share information more frequently as time elapses $(\beta=2.92, p<0.001)$, whereas those in Group 2 primarily share information early in the mission $(\beta=-3.12, p<0.001)$.

\section{Discussion and Conclusions}

This study contributes to the literature on information sharing in a virtual team context in a number of ways. First, we adopt a temporal approach to studying the information sharing process. While prior literature has implicitly acknowledged the processual nature of transmitting information, no study to our knowledge has focused on specific sharing events. Thus, our emphasis on discrete sharing actions provides a new perspective on this process. Second, we delineate a number of behavioral and cognitive factors that may influence the decision to share information. These effects are based on the prior sequence of communication events, sharing events, and the current team state. Though these factors may not all be salient to individuals (e.g. they may not accurately identify central team members), we demonstrated empirically that there are distinct trends in sharing behavior, and nearly all of the identified factors played a role in shaping decisions. Finally, we make a broader methodological contribution with the application of a latent-class relational event model. This method allows us to not only infer patterns in sequence data, but also to cluster individuals into groups based on commonalities in their decision-making tendencies.

Our dynamic, event-based approach is a natural extension of prior work on expertise coordination and knowledge sharing in virtual teams and online communities. Indeed, much of the extant literature emphasizes the role of interactions in shaping 
coordination [17], [23], [33]. However, in these studies interactions tend to be compressed into static network configurations c.f. [32], [33], thereby losing the granularity of data at the event level see [48]. Like coordination and collaboration, information sharing is a process composed of interactions between team members that unfold over time. Thus, we applied a framework that explicitly focuses on events and the temporal relationships between events in a sequence. This shift allows us to focus on when and why an action took place, and allows us to differentiate sequences, even if they start and end at the same point. For example, two individuals may share the same amount of information with the same people. However, one of these two conducts all of their sharing before establishing a relationship through informal communication, while the other builds strong ties first. These two patterns of behavior are distinct, however they could not be captured by a purely compositional or configural perspective [46].

Our empirical analysis uncovered two distinct categories of decision-making behavior. In essence, each individual's set of decisions could be categorized into one of two broad archetypes, accounting for individual heterogeneity. Between these two classes we observe significant differences in behavioral tendencies. The first category is defined by sharing information later in the mission and at points in time when they are central in the network. Essentially, these individuals spend time communicating first, and become proportionately more active than other team members. As they become relatively more focal, they accelerate their rate of transmitting information, and they tend to select individuals who have not communicated frequently. These individuals also tend to send more information to others with whom they have shared previously, thus building strong individual pathways. On the other hand, the second category is defined by sharing information early, and transmitting that information to active individuals in the informal communication network. These individuals tend to share more when they play a less active role in communicating with others, suggesting they substitute the two behaviors.

\subsection{Implications for Team Functioning}

Our findings also provide some insight into team functioning and group behavior. First, the patterns we observed emerged from ad hoc teams with no background working together. Participants in the study did not have significant time to plan and execute a strategy, nor could they complete the task multiple times. Thus, the resulting behaviors were purely organic, and emerged out of natural tendencies. This finding has implications for online communities or open source platforms where individuals work as a team, but are not formed and controlled by any formal mechanisms [25]. A stakeholder could use these patterns to gain some intuition into how contributors may share information or coordinate expertise, and could attempt to manage or influence those patterns.

Second, the two classes we identified may be more or less effective in a variety of contexts. In fastresponse teams for example, team members need to act quickly to address a crisis or other pressing deadline [23], [24]. Under these conditions, individuals who follow the second set of decision rules may be more effective, given their propensity to share early. On the other hand, for longer-duration tasks or situations where information sharing is costly, it may be beneficial for individuals to establish a focal role in the team before sharing information. Essentially, the development of meta-knowledge would be critical to team effectiveness. In this scenario, the first decision-making approach may be ideal. Thus, a team leader or facilitator can determine what type of task their group is facing and what the constraints are; with this knowledge, they may then influence or incentivize certain tendencies, leading to improved performance.

\subsection{Limitations}

We do acknowledge some limitations to our study that potentially limit the generalizability of our research to practice. First, the virtual teams were made up of students, and these participants had no previous experience working with one another, and had no expectation of working together in the future. These sessions had no interruptions or external influences for two hours. These conditions are not realistic in most work environments. However, there are also advantages: the laboratory setting allowed us to specifically track the sharing of tangible intelligence in real time and in relation to communication behavior. Further, we were able to observe fully emergent patterns in an ad hoc setting. Future research should extend these findings to actual organizations. Second, while we did identify the informational content of each message, we did not account for other semantic qualities such as affect or tone. To the extent that we were able, we attempted to filter out references to information that were superfluous, such as inquiring about the state of a cell or checking to see if the obstacle has been cleared. These interactions, though they contained information, are not sharing in the context of what we are analyzing. Semantic content could also provide insight into why redundant information is shared, to what extent an information strategy was planned, or if there was an active search that precipitated the transmittance of information. While we believe that this analysis would 
be a fruitful direction for future research, it is beyond the scope of this study.

Finally, this study did not consider the implications of the two behavioral profiles for performance. Indeed, it is likely that one group would be better than another with regards to metrics such as accuracy or speed. Future work should include post hoc analyses of various performance metrics to identify the implications of the different behaviors.

\section{References}

[1] M. Alavi and D. E. Leidner, "Review: Knowledge management and knowledge management systems: Conceptual foundations and research issues,” MIS Q., pp. 107-136, 2001.

[2] R. L. Daft and R. H. Lengel, "Organizational information requirements, media richness and structural design,” Manag. Sci., vol. 32, no. 5, pp. 554571, 1986.

[3] I. Nonaka, “A dynamic theory of organizational knowledge creation,” Organ. Sci., vol. 5, no. 1, pp. 1437, 1994.

[4] L. P. Robert, A. R. Dennis, and M. K. Ahuja, "Social capital and knowledge integration in digitally enabled teams,” Inf. Syst. Res., vol. 19, no. 3, pp. 314334, 2008.

[5] S. W. J. Kozlowski and B. S. Bell, Work groups and teams in organizations, vol. 12. New York: Wiley, 2003.

[6] J. R. Mesmer-Magnus and L. A. DeChurch, "Information sharing and team performance: a metaanalysis,” J. Appl. Psychol., vol. 94, no. 2, p. 535, 2009.

[7] J. R. Mesmer-Magnus, L. A. DeChurch, M. Jimenez-Rodriguez, J. Wildman, and M. Shuffler, “A meta-analytic investigation of virtuality and information sharing in teams," Organ. Behav. Hum. Decis. Process., vol. 115, no. 2, pp. 214-225, 2011. [8] D. S. Staples and J. Webster, "Exploring the effects of trust, task interdependence and virtualness on knowledge sharing in teams,” Inf. Syst. J., vol. 18, no. 6, pp. 617-640, 2008.

[9] A. Tiwana and E. R. Mclean, "Expertise integration and creativity in information systems development," J. Manag. Inf. Syst., vol. 22, no. 1, pp. 13-43, 2005. [10] J. Shore, E. Bernstein, and D. Lazer, "Facts and figuring: An experimental investigation of network structure and performance in information and solution spaces,” Organ. Sci., vol. 26, no. 5, pp. 1432-1446, 2015.

[11] S. Y. Choi, H. Lee, and Y. Yoo, "The impact of information technology and transactive memory systems on knowledge sharing, application, and team performance: a field study,” MIS Q., pp. 855-870, 2010.

[12] P. Kanawattanachai and Y. Yoo, “The impact of knowledge coordination on virtual team performance over time,” MIS Q., pp. 783-808, 2007.

[13] P. M. Leonardi, “Ambient Awareness and Knowledge Acquisition: Using Social Media to Learn" Who Knows What" and" Who Knows Whom",’ MIS Q., vol. 39, no. 4, pp. 747-762, 2015.

[14] A. M. Carton and J. N. Cummings, "A theory of subgroups in work teams,” Acad. Manage. Rev., vol. 37, no. 3, pp. 441-470, 2012.

[15] W. Tsai, "Social structure of ‘coopetition' within a multiunit organization: Coordination, competition, and intraorganizational knowledge sharing," Organ. Sci., vol. 13, no. 2, pp. 179-190, 2002.

[16] C. B. Gibson and J. L. Gibbs, "Unpacking the concept of virtuality: The effects of geographic dispersion, electronic dependence, dynamic structure, and national diversity on team innovation,” Adm. Sci. Q., vol. 51, no. 3, pp. 451-495, 2006.

[17] S. Faraj and L. Sproull, “Coordinating Expertise in Software Development Teams,” Manag. Sci., vol. 46, no. 12, pp. 1554-1568, 2000.

[18] M. S. Poole, "On the Study of Process in Communication Research,” in Communication Yearbook 36, 2012, p. 371.

[19] A. H. Van de Ven and M. S. Poole, “Alternative approaches for studying organizational change,” Organ. Stud., vol. 26, no. 9, pp. 1377-1404, 2005. [20] E. Quintane and G. Carnabuci, "How Do Brokers Broker? Tertius Gaudens, Tertius Iungens, and the Temporality of Structural Holes,” Organ. Sci., 2016. [21] C. DuBois, C. T. Butts, D. McFarland, and P. Smyth, "Hierarchical models for relational event sequences,” J. Math. Psychol., vol. 57, no. 6, pp. 297309, 2013.

[22] C. DuBois, C. T. Butts, and P. Smyth, "Stochastic blockmodeling of relational event dynamics,"

presented at the International Conference on Artificial Intelligence and Statistics, 2013, vol. 31, pp. 238-246. [23] S. Faraj and Y. Xiao, "Coordination in FastResponse Organizations,” Manag. Sci., vol. 52, no. 8, pp. 1155-1169, 2006.

[24] A. Majchrzak, S. L. Jarvenpaa, and A. B. Hollingshead, "Coordinating Expertise among Emergent Groups Responding to Disasters,” Organ. Sci., vol. 18, no. 1, pp. 147-161, 2007.

[25] A. Majchrzak and A. Malhotra, "Effect of Knowledge-Sharing Trajectories on Innovative Outcomes in Temporary Online Crowds,” Inf. Syst. Res., vol. 27, no. 4, pp. 685-703, Nov. 2016. [26] G.-W. Bock, R. W. Zmud, Y.-G. Kim, and J.-N. Lee, "Behavioral intention formation in knowledge sharing: Examining the roles of extrinsic motivators, 
social-psychological forces, and organizational climate,” MIS Q., pp. 87-111, 2005.

[27] C.-M. Chiu, M.-H. Hsu, and E. T. Wang, "Understanding knowledge sharing in virtual communities: An integration of social capital and social cognitive theories,” Decis. Support Syst., vol. 42, no. 3, pp. 1872-1888, 2006.

[28] A. Kankanhalli, B. C. Tan, and K.-K. Wei, "Contributing knowledge to electronic knowledge repositories: an empirical investigation,” MIS Q., pp. 113-143, 2005.

[29] M. M. Wasko and S. Faraj, "Why should I share? Examining social capital and knowledge contribution in electronic networks of practice,” MIS Q., pp. 35-57, 2005.

[30] S. Faraj and S. L. Johnson, "Network exchange patterns in online communities,” Organ. Sci., vol. 22, no. 6, pp. 1464-1480, 2011.

[31] M. M. Wasko and S. Faraj, “'It is what one does': why people participate and help others in electronic communities of practice,” J. Strateg. Inf. Syst., vol. 9, no. 2, pp. 155-173, 2000.

[32] S. Kudaravalli and S. Faraj, "The Structure of Collaboration in Electronic Networks,” J. Assoc. Inf. Syst., vol. 9, no. 10/11, pp. 706-726, Oct. 2008.

[33] S. Kudaravalli, S. Faraj, and S. L. Johnson, “A configural approach to coordinating expertise in software development teams,” MIS Q., vol. 41, no. 1, pp. 43-64, 2017.

[34] M. E. Sosa, M. Gargiulo, and C. Rowles, "Can informal communication networks disrupt coordination in new product development projects?,” Organ. Sci., vol. 26, no. 4, pp. 1059-1078, 2015.

[35] R. Leenders, N. Contractor, and L. DeChurch, "Once upon a time: Understanding team processes as relational event networks,” Organ. Psychol. Rev., vol. 1, no. 24, 2015.

[36] J. Gaskin, N. Berente, K. Lyytinen, and Y. Yoo, "Toward Generalizable Sociomaterial Inquiry: A Computational Approach for Zooming In and Out of Sociomaterial Routines,” MIS Q., vol. 38, no. 3, pp. 849-871, 2014.
[37] W. Chen, X. Wei, and K. X. Zhu, “Engaging voluntary contributions in online communities: A hidden Markov model,” MIS Q., vol. 42, no. 1, 2017. [38] P. V. Singh, Y. Tan, and N. Youn, “A hidden Markov model of developer learning dynamics in open source software projects," Inf. Syst. Res., vol. 22, no. 4, pp. 790-807, 2011.

[39] R. Reagans and B. McEvily, "Network structure and knowledge transfer: The effects of cohesion and range,” Adm. Sci. Q., vol. 48, no. 2, pp. 240-267, 2003. [40] M. T. Hansen, M. L. Mors, and B. Løvås, "Knowledge sharing in organizations: Multiple networks, multiple phases,” Acad. Manage. J., vol. 48, no. 5, pp. 776-793, 2005.

[41] M. T. Hansen, "Knowledge networks: Explaining effective knowledge sharing in multiunit companies,” Organ. Sci., vol. 13, no. 3, pp. 232-248, 2002.

[42] M. Granovetter, “The strength of weak ties,” Am. J. Sociol., vol. 78, no. 6, pp. 1360-1380, 1973. [43] S. L. Johnson, S. Faraj, and S. Kudaravalli, "Emergence of Power Laws in Online Communities: The Role of Social Mechanisms and Preferential Attachment,” MIS Q., vol. 38, no. 3, pp. 795-808, 2014.

[44] C. T. Butts, “A relational event framework for social action,” Sociol. Methodol., vol. 38, no. 1, pp. 155-200, 2008.

[45] C. T. Butts and C. S. Marcum, "A Relational Event Approach to Modeling Behavioral Dynamics,” in Group Processes, Springer International Publishing, 2017, pp. 51-92.

[46] A. Schecter, A. Pilny, A. Leung, M. S. Poole, and N. Contractor, "Step by step: Capturing the dynamics of work team process through relational event sequences,” J. Organ. Behav., 2017.

[47] C. DuBois and P. Smyth, "Modeling relational events via latent classes," presented at the Proceedings of the 16th ACM SIGKDD international conference on Knowledge discovery and data mining, 2010, pp. 803812.

[48] E. Quintane, G. Conaldi, M. Tonellato, and A. Lomi, "Modeling Relational Events A Case Study on an Open Source Software Project,” Organ. Res.

Methods, vol. 17, no. 1, pp. 23-50, 2014. 